\title{
Experimental Validation Of An Innovative Procedure For The Rolling Noise Correction
}

\author{
Massimo Viscardi ${ }^{*}$, Pasquale Napolitano ${ }^{1}$, Stefano Ferraiuolo ${ }^{2}$ \\ ${ }^{1}$ University of Naples "Federico II", Dept. of Industrial Engineering, Via Claudio, 21 - 80125 Napoli - Italy \\ ${ }^{2}$ HITACHI RAIL - Via Argine, 425 - 80147, Napoli - Italy
}

\begin{abstract}
Among the wide contest of the train vehicles rolling noise evaluation, the aim of the paper is the development, implementation and experimental testing of a new method for roughness calculation according to FprCEN/TR 16891:2015 and the successive evaluation of the correction parameters of the measured rolling noise due to the presence of not compliant rail roughness. It is, in-fact, a very often operative condition, the execution of rolling noise tests over standard in-operation rails that are characterized by roughness profiles very different from standard one as those prescribed within the ISO 3095 procedure. Very often, this difference lead to the presence of an exceeding noise that needs to be evaluated and revised for a correct definition of the phenomena. Within the paper, the procedure implementation is presented and later on verified in operative experimental contest; forecasted and measured data are compared and successively commented.
\end{abstract}

\section{GENERAL CONTEST OF THE RESEARCH}

In railway sector noise is a really relevant problem. Often trains, especially low speed trains, walk trough cities or near houses. For this reason the noise produced by trains must be kept under control. The maximum admissible Sound Pressure Level (SPL) is the value frequently constrained and the norms, in last years, has shown a decreasing trend for maximum admissible value. According to this trend manufacturers have developed ever better method for noise control and reduction.

In railway sector there are three relevant noise sources; they are listed below:

- $\quad$ Traction Noise;

- Aerodynamic Noise;

- $\quad$ Rolling Noise;

and the noise, as function of speed, is shown in Figure 1.

For low speed trains, less than $100 \mathrm{kph}$, the aerodynamic noise can be neglected and the most relevant sources are the traction noise, almost constant with speed variation, and rolling noise that is linearly increasing, more or less, according to speed. The traction noise and aerodynamic noise can be easy kept under control; the first one using a more quite engine or a best insulation engine bay; the second one depends, in first approximation, by the shape of frontal area of the train.

Rolling noise generation is more complex. In that process are involved two main components:

- Wheel;

- Rail;
In most country, Italy is one of them, company in charge for wheel is not in charge for rail and vice versa.

When the noise generated by wheel/rail interaction is higher than maximum admissible level it is hard to identify clearly if it is due to wheel or rail.

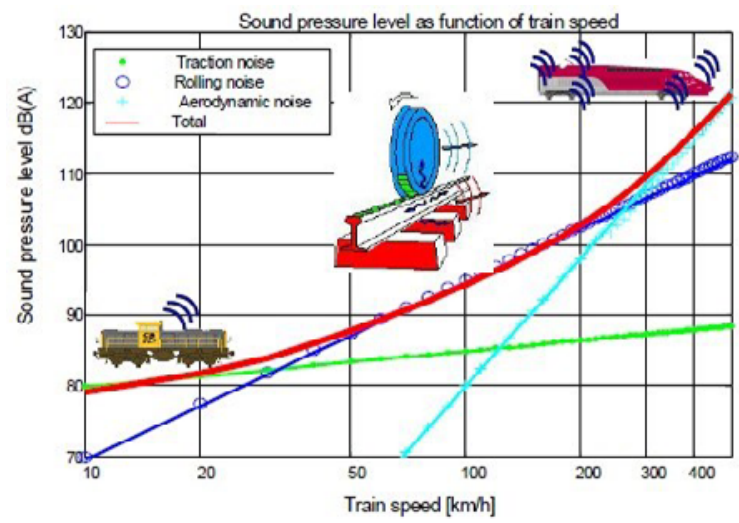

Figure 1 : Sources SPL vs. speed.

An example of rolling noise spectrum is given in Figure 2. To make general background more complex the company in charge for rail is the one who establish the maximum allowable value for rolling noise SPL. In this situation it is important for customer have a procedure and a tool to split rolling noise between rail and wheel.

In previous work [7] a computational tool that, according to UNI EN ISO FPRCEN/TR,16891 [6] can correct, eliminating it, the contribute of rail to rolling noise has been preliminary developed and implemented.

*Corresponding author: massimo.viscardi@unina.it 


\section{THEORETICAL BACKGROUND}

There are four mechanisms that are suggested in the literature, as the main causes of rolling noise.

These are:

- Rail and wheel roughness,

- Parameter variation, or moduli heterogeneity,

- Creep,

- Aerodynamic noise.

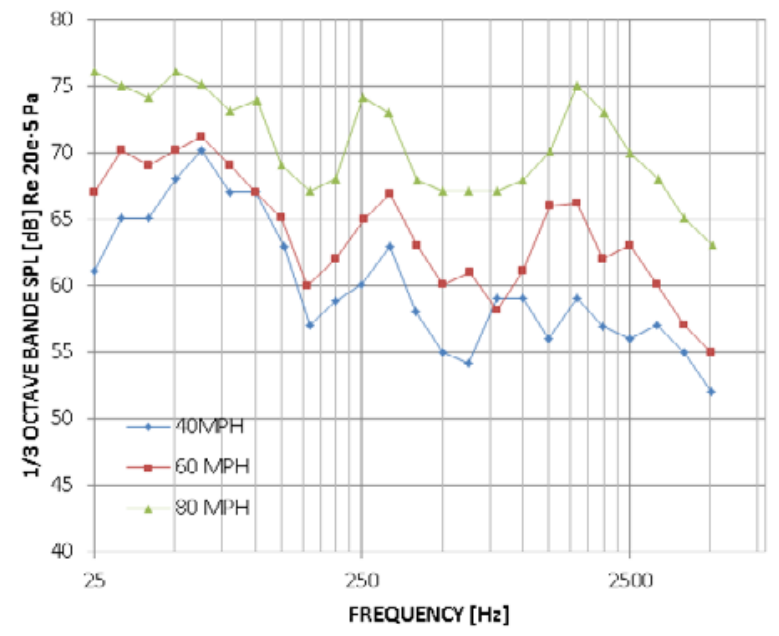

Figure 2 : Pass-by noise for single car vehicle with aluminum centered wheels on ballast and tie track.

\subsection{Wheel/Rail Roughness}

Is probably the most significant cause of the wheel/rail noise. The surface roughness profile may be decomposed into a continuous spectrum of wavelengths. At wavelengths short relative to the contact patch dimension, the surface roughness is attenuated by averaging of the roughness across the contact patch, an effect which is described as contact patch filtering. Thus, fine regular grinding marks of dimensions less than, perhaps, $1.5 \mathrm{~mm}$ should not produce significant noise compared to lower frequency components.

\subsection{Parameter Variation}

Parameter variation refers to the variation of rail and wheel steel moduli, rail support stiffness, and contact stiffness due to variation in rail head transverse radiusof-curvature. The influence of fractional changes in elastic moduli and of radius-of-curvature of the rail head as a function of wavelength necessary to generate wheel/rail noise equivalent to that generated by surface roughness is illustrated in the figure. Experimental data for the effect of modulus variation at this frequency have not yet been found. Rail head ball radius heterogeneity also induces a dynamic response in the wheel and rail. The variation of rail head curvature would have to be -on the order of $10 \%$ to $50 \%$ to produce a noise level similar to that produced by rail roughness alone. Data on rail head radii of curvature as a function of wavelength have not been obtained nor correlated with wayside noise. Also, rail head ball radius variation will normally accompany surface roughness, so that distinguishing between ball radius variation and roughness may be difficult in practice.

\subsection{Dynamic Creep}

Dynamic creep may include both longitudinal and lateral dynamic creep, roll-slip in a direction parallel with the rail, and spin-creep of the wheel about a vertical axis normal to the wheel/rail contact area.

\subsubsection{Longitudinal Creep}

It is not considered significant by some researchers, as rolling noise levels are claimed to not increase significantly during braking or acceleration on smooth ground rail. However, qualitative changes of the sound of wheel/rail noise on newly ground rail with a grinding pattern in the rail running surface is observable to the ear as a train accelerates or decelerates, in contradiction to the notion that longitudinal creep is of no significance.

\subsubsection{Lateral creep}

It occurs during curve negotiation, and is responsible for the well-known wheel squeal phenomena resulting from stick-slip. Lateral creep may not be significant at tangent track, but lateral dynamic creep may occur during unloading cycles at high frequencies on abnormally rough or corrugated rail. Lateral dynamic creep is postulated by some to be responsible for short-pitch corrugation at tangent track. Therefore, lateral creep, at least in the broad sense, may be a significant source of noise.

\subsubsection{Spin-creep}

It is caused by wheel taper which produces a rolling radius differential between the field and gauge sides of the contact patch.

\subsection{Aerodynamic Noise}

Aerodynamic noise is caused by turbulent boundary layer noise about the wheel circumference as it moves forward and by under car components which exhibit substantial aerodynamic roughness. Noise due to air turbulence about the wheel is usually not significant at train speeds representative of transit systems, while noise due to air turbulence in the truck area may be significant.

Among these possible cause, the one that generally requires more attention is the roughness where/rail roughness and, if referred to the acceptance test to be performed at the train delivery from the manufacture to the customer, mainly the rail roughness as the wheel is 
generally considered as a smooth profile at this operational stage.

During these acceptance test, pass-by acoustic measurements are generally performed at different speeds and train is generally required to not exceed limit noise level at specific measurement station (generally 7,5 $\mathrm{m} @ 1,2 \mathrm{~m}$ height form the centerline). Measurement site is always a straight portion of track line, that is the reason why other phenomena are generally negligible and only rail roughness may strongly influence the measured noise level.

ISO 3095 prescribe the limit roughness to be acceptable during the test performance, but very often these strong requirements cannot be satisfied because real portion of in service tracks are used for test and rectification process are extremely expensive and time consuming as well require the stop of service operation along the train line.

Hence the reason for a correct evaluation of rail roughness and the implementation of a correction procedure [7].

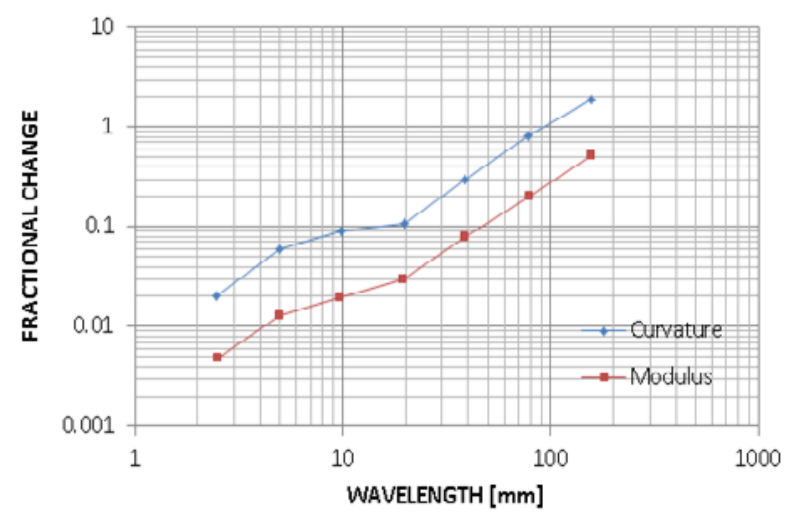

Figure 3 : Change in elastic modules and railhead curvature required to generate wheel/rail excitation.

\section{Test Specimen Definition}

Along this work, a medium weight metro passenger vehicle has been considered. The vehicle present the following main characteristics:

- Composition: M-R-M+M-R-M;

- Max Length: approx. 110 meters;

- Max Width: approx. 3 meters;

- $\quad$ Structure Material: Aluminum;

- $\quad$ Seats: approx. 200;

- $\quad$ Stand up passengers: approx. 1000;

- $\quad$ Maximum Speed: $90 \mathrm{kph}$;

Vehicle profile is presented in the following figure 4 and 5.

In real operative conditions a wide list of configuration are available for the train according to different equipment status. To answer at reference technical specifications, the test and relative data post processing and comparison of a single condition has been identified as standard condition. This operative condition presented:

- HVAC: on;
- Traction System: on;

- Auxiliary Inverter: on;

- Engine and Reducer: on;

- Compressor: on;

- $\quad$ speed: $60 \mathrm{kph}$;

This condition will be used for all tests showed in this paper and will be reference condition for data post processing and comparison.

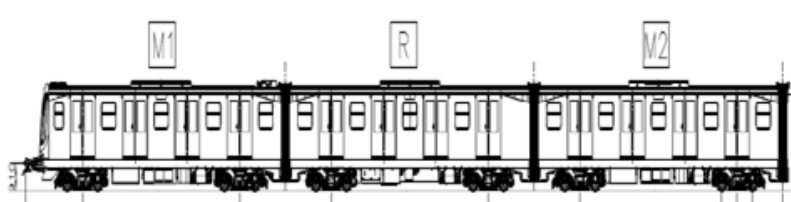

Figure 4 : Side view of half vehicle

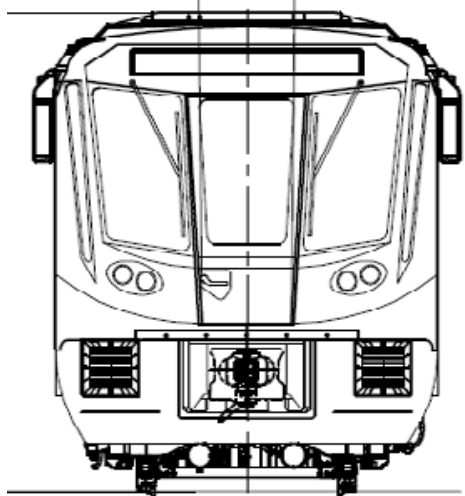

Figure 5 : Front view

\section{General Test Description}

The test site was located near in Italy and it was accurately chosen to best fulfill the standard requirements. In the specific, two factors have been considered:

- $\quad$ Straight configuration of the track

- No reflecting surfaces near the measurement point

\subsection{Rail Conditions}

According to the UNI EN ISO 3381/3095 the rail must have a maximum roughness, for third band octave, less than a threshold value. This requirement is really important for noise tests acceptance. In Figure 6 the standard roughness curve according to UNI EN ISO 3095_2005.

As said before the roughness spectrum of rail was not known at test moment and its calculation was one of the scope of the tests.

\subsection{Vehicle Conditions}

During the test the train was considered empty, no passenger on board except people necessary for text execution. The wheel was in good condition and nor 
fatigue or excessive roughness was observed. During tests all the train equipment were settled as specified in chapter 3.

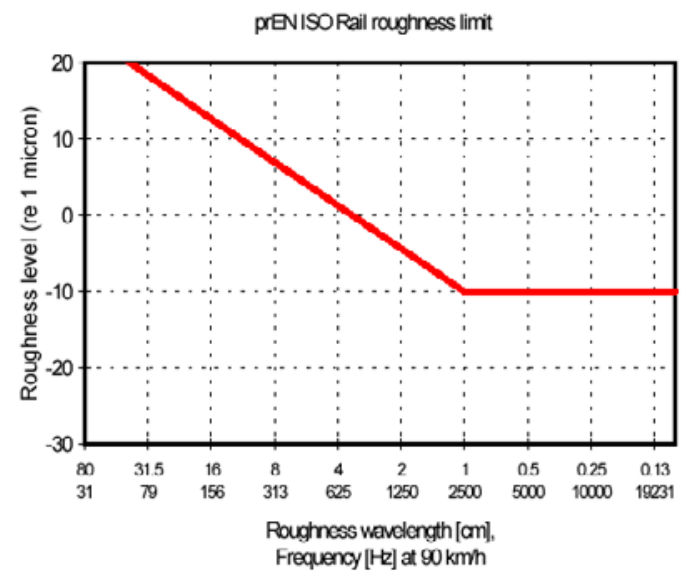

Figure 6 : Limit Roughness spectra

\section{Acceleration Test Description}

The acceleration was measured during multiple train passby to obtain a mean value of acceleration. For this test all general condition expressed in chapter 4 are applied to obtain a general standard result that can be compare with other tests. For accelerometer measurements a set of accelerometers was used. According to reference norm they were place on the railway is appropriate positions. As shown in Figure 7 the accelerometer position is defined by norm and have been repeated on experimental test as shown in Figure 8. Acceleration spectra along the $\mathrm{z}$ axis will be the basic input for the track decay calculation [6-7]; with acceleration along $\mathrm{y}$, with a similar process, the longitudinal decay rate may be calculated. Because this parameter is less useful, it will be not presented within this paper.

In this specific test, three tri-axial accelerometers have been used also to avoid problem related to the need of compensating profiles. Also, an extra accelerometer has been positioned on the rail web for further consideration; it has not been used for the purposes of the work.

\subsection{Acceleration Result}

According to train configuration (see chapter 3), to test environment specification (see chapter 4 ) and accelerometer position (see chapter 5) shown above the acceleration has been measured for all accelerometers for more than one passage.

An acceleration example in shown in Figure 9 where a typical passage is plotted as function of time.

For decay rate calculation only vertical acceleration will be considered and only decay rate used for roughness calculation.

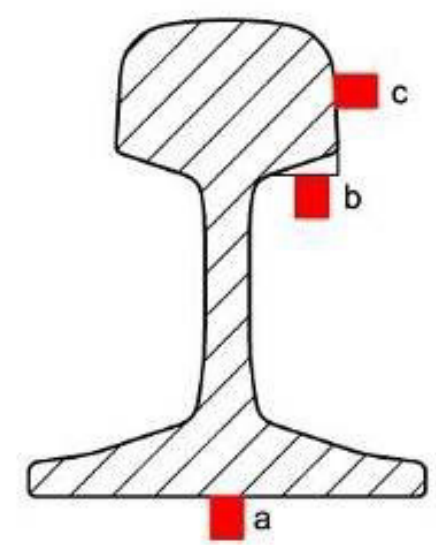

Figure 7 : Norm suggested points

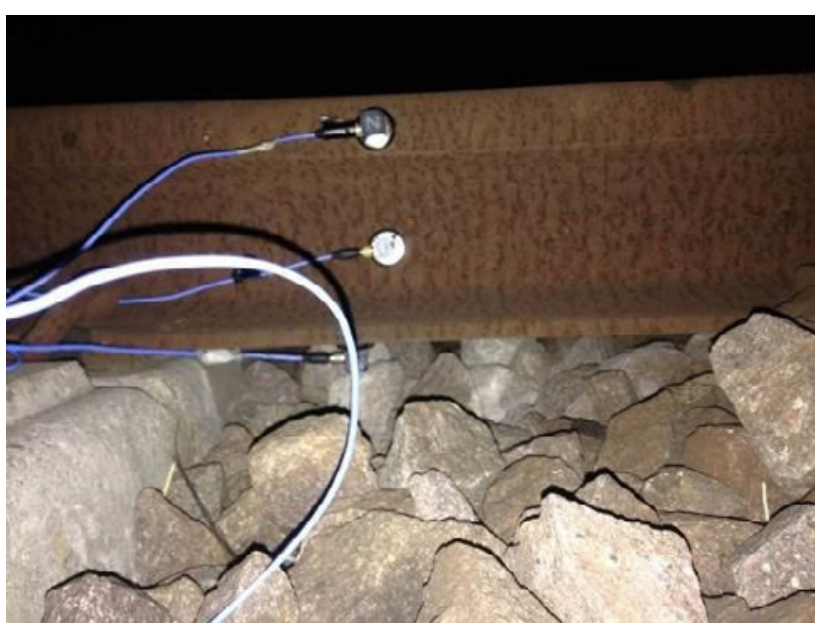

Figure 8: Accelerometers positioned on the rail

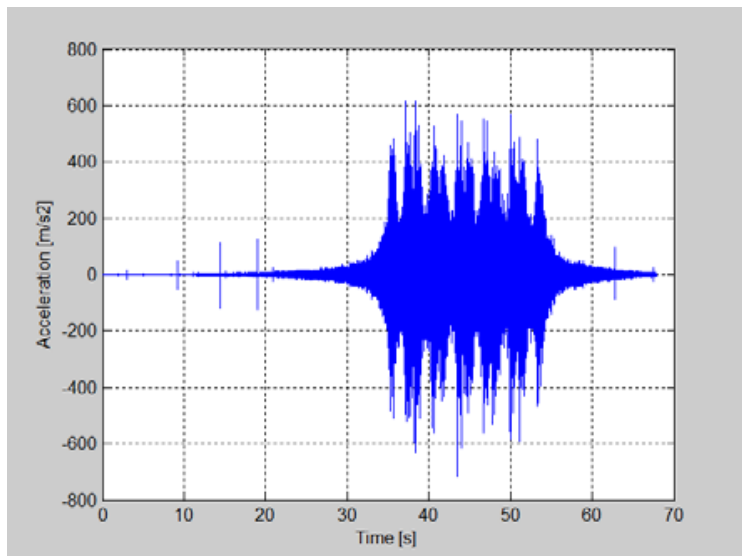

Figure 9 : Time history spectrum for single passage. Refers to accelerometer position $\mathrm{C}$ in reference norme

\section{Roughness Calculation}

Once that acceleration has been measured, according to normative the first step for roughness calculation is the decay rate calculation that depends from the FRF of the system. The FRF has been calculate on whole train passage and around a single wheel passage. The difference between two are shown in figure 10 and 11 . 


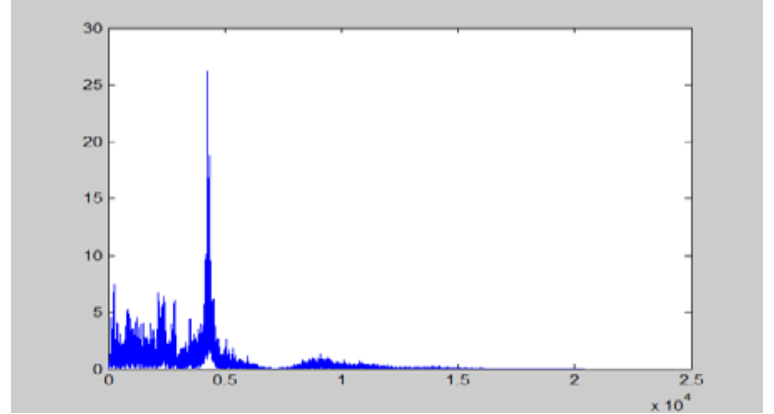

Figure 10 : FRF on whole passage

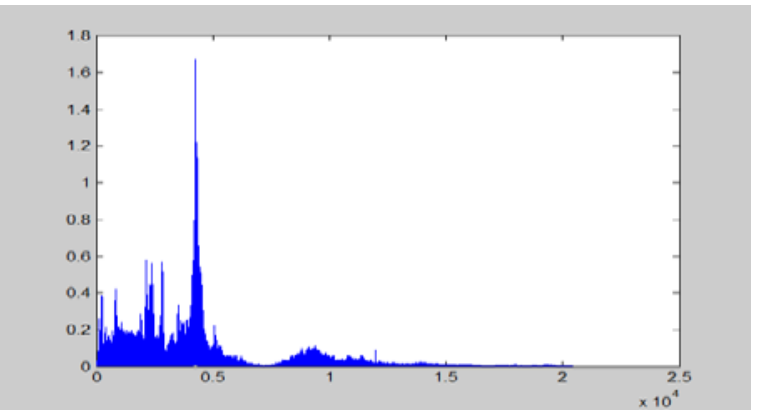

Figure 11 : FRF referred at a single wheel

Using the iterative method and a residual error of 10 5 the decay rate is than calculate for a wide number of measures as shown in Figure 12.

The mean value of decay rate is than used for roughness calculation.

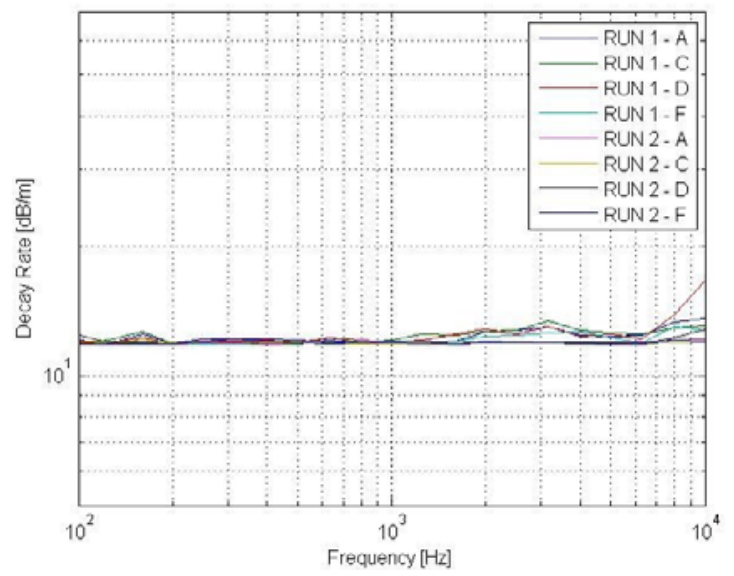

Figure 12 : Decay rate for several measures

The calculated roughness, if compared with the threshold level of Figure 6, has a really relevant value and cannot be neglect during noise tests.

As prescribed within the ISO 3095, the excess roughness need in-fact to be corrected by the use of a specific procedure that will introduced in the following chapter 8 .

\section{Acoustic Tests Description}

Acoustic test were performed according to the pass-by noise reference EN ISO 3095. For every train passage the SPL was measured at 7.5 meters away from the center of railway seat in orthogonal direction and at 1.2 meters from ground.

The test speed was fixed at $60 \mathrm{Km} / \mathrm{h}$ according to the technical requirement even if measurement were also performed at different speed for research purposes (from $20 \mathrm{Km} / \mathrm{h}$ to $60 \mathrm{Km} / \mathrm{h}$ step $10 \mathrm{Km} / \mathrm{h}$ ).

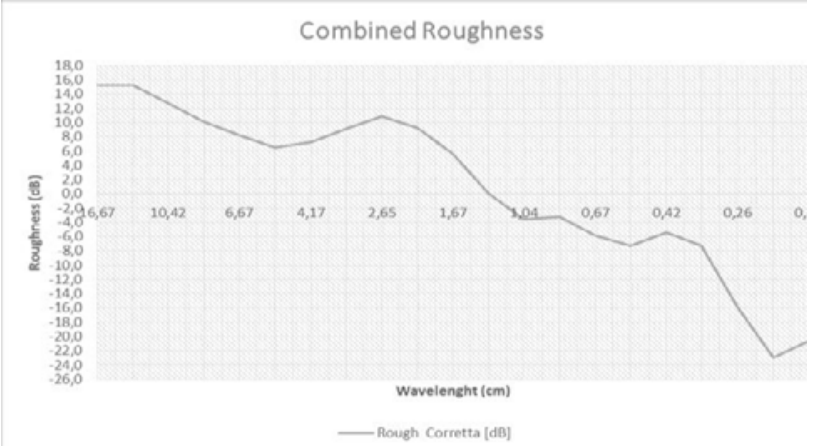

Figure 13 : Combined roughness calculated

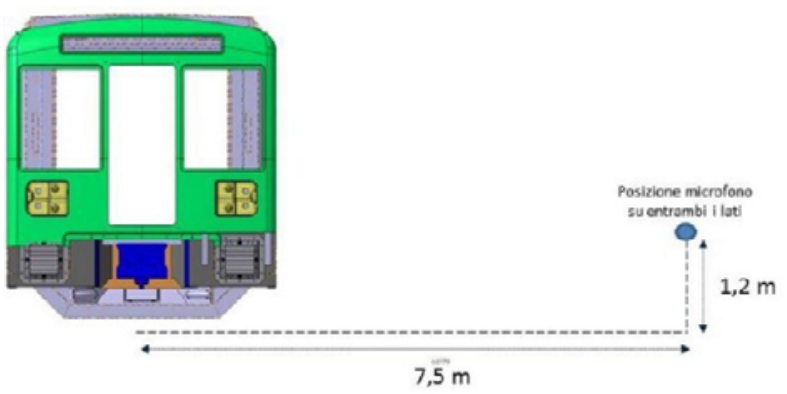

Figure 14 : Noise test scheme

\subsection{Noise Measurements Results}

In the following pictures, some typical results are reported. Figure 15 show the typical pass-by noise profile; also the pressure fluctuation in correspondence of the single boogie axles is evident if a zoom on the maximum levels is performed (figure16).

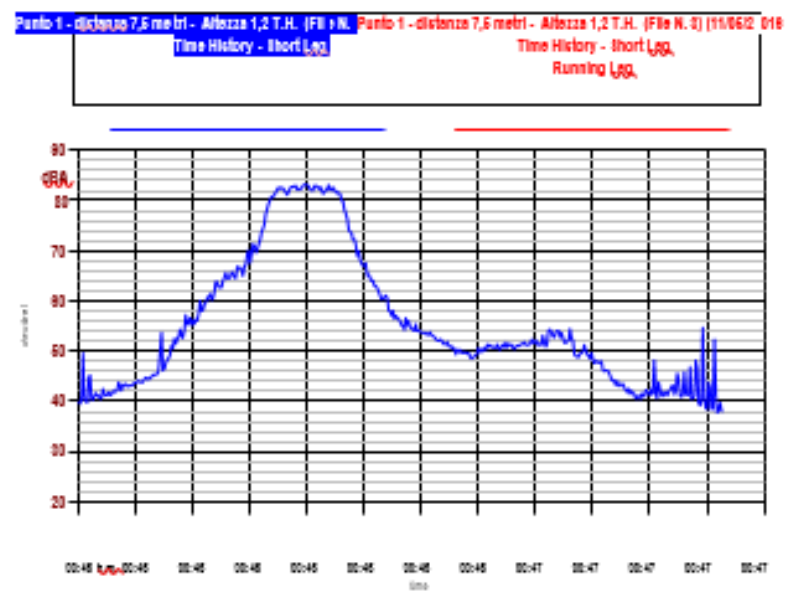

Figure 15 : Time history typical passage

Data show an equivalent level over the passage (intended as the time window when the train pass through the measurement station that is approximately 6 seconds for the specific speed) Laeq of about $82.3 \mathrm{~dB}(\mathrm{~A})$. 
This value has been measured over 6 passage with a difference among them of less than $0,5 \mathrm{~dB}(\mathrm{~A})$. Within the next pictures, the Equivalent and maximum noise spectra are presented.

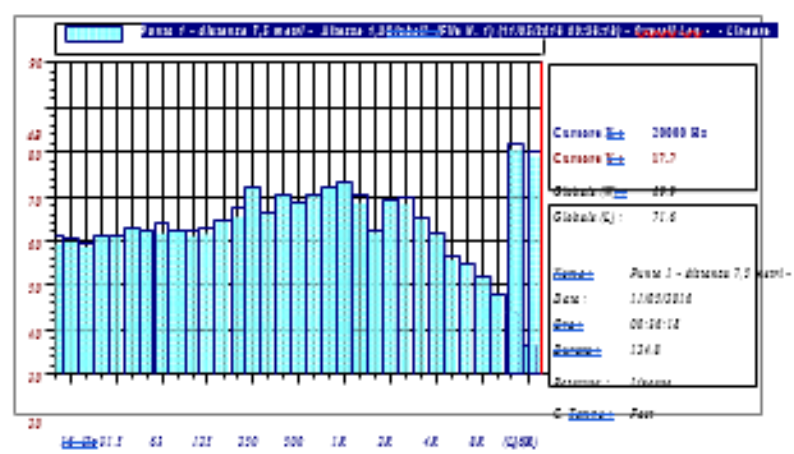

Figure 16 : Leq for typical passage

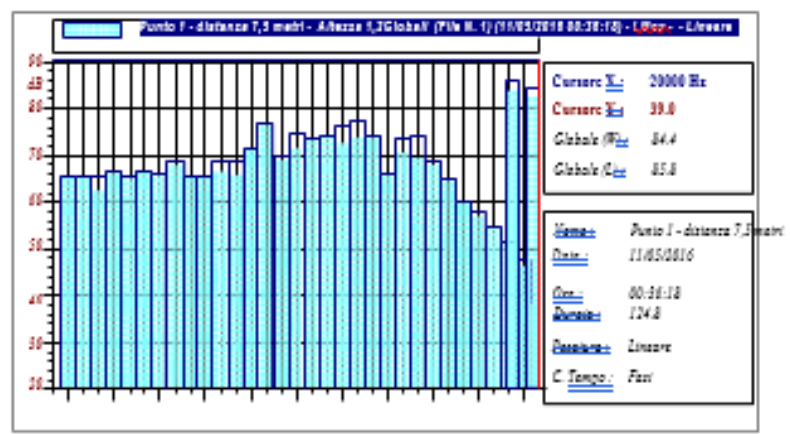

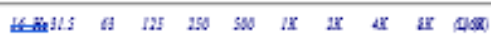

Figure 17 : Leq for typical passage

\section{Noise Correction Procedure}

The noise correction procedure, involves three steps. The first one is the FRF calculation to relate the noise measured to the actual roughness (to allow this passage the system will be considered linear).

The second step consist in the new roughness calculation. According to norm the noise should be measured when the roughness is less or equal at threshold roughness. The actual roughness over come the limit one and needs to be compensated.

The third step consist in noise evaluation and will involve the FRF and the roughness calculated in step one and two.

\subsection{FRF Calculation}

The FRF will be estimated according to following equation

$$
\begin{aligned}
L_{\text {RpRt ot,nt }}\left(f_{c}\right) & =L_{p e q, t p}\left(f_{c}\right)-L_{R t o t}\left(f_{c}, v\right) \\
& -10 \lg \left(\frac{N_{a x}}{l}\right)
\end{aligned}
$$

where LHpRtot,nl(fc ) is the FRF we want estimate, Lpeq,tp the measured noise, LRtot the total roughness, Nax the number of wheels and 1 the train length.
The total transfer function $L_{-}($HpRtot,nl $)\left(f_{c}\right)$ is independent from the roughness, train length and number of axles. It characterizes the vibro-acoustic properties of the vehicle, the track and the propagation area.

The FRF result is shown in Figure 18.

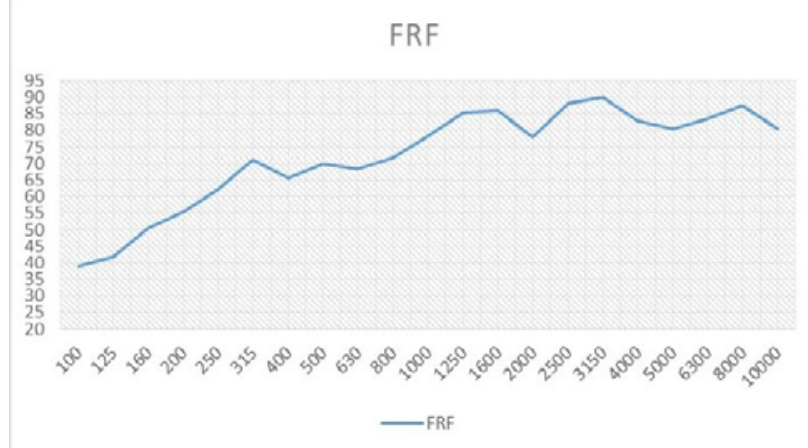

Figure 18 : Estimated FRF

\subsection{Roughness Correction}

The roughness is corrected according to UNI EN ISO 3095. For UNI EN ISO 3095 two revision are available. The first one from 2005 and the second one from 2013. The two curves, as shown in Figure 19, are slightly different. According to literature the most relevant zone for rolling noise generation is between $0.033[\mathrm{~m}]$ and $0.010[\mathrm{~m}]$ and in this range the curves are approximately the same. In any case the most recent curve will be used as reference.

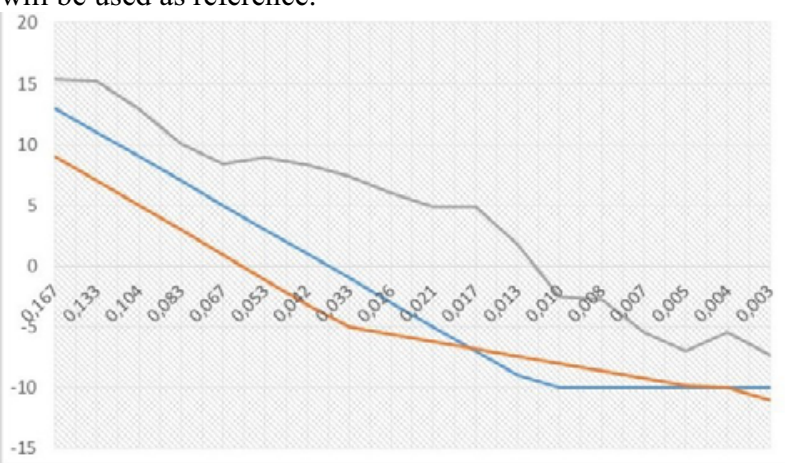

Figure 19: Calculated roughness (grey), UNI EN ISO 3095:2005 (red) and UNI EN ISO 3095:2013 (blue) comparison versus wavelength domain

\subsection{Correct Noise Evaluation}

The correct noise evaluation will be done according to [7] and simply resolving Equation 1 respect to the SPL. According to results shown up to now the correct noise, compared to measured noise, is shown in Figure 20.

\section{Confronto SPL dB(A)}

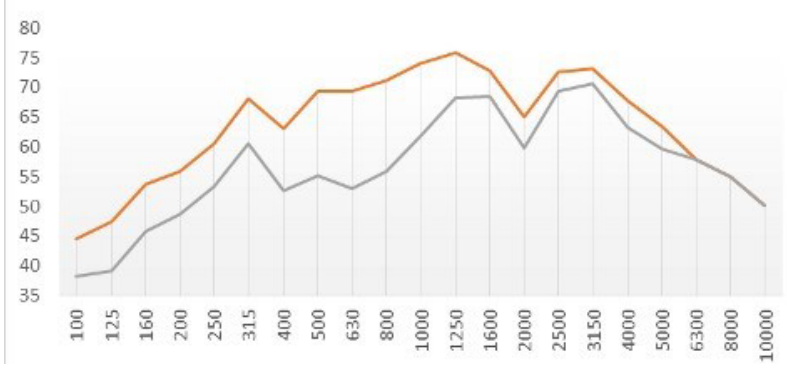

Figure 20 : Original noise (overall level 82.4 [dBA]) and corrected noise (overall $75.9[\mathrm{dBA}]$ ) comparison 


\section{Conclusions}

As shown in this paper the rolling noise is a relevant problem for train sector and must be taken under control. In the first part of the paper it has been underlined that not compliant rail conditions, with presence of severe roughness profiles may lead to exceeding noise level.

In the second part of paper an innovative procedure for exceeding rail roughness has been proposed and experimented. A dedicated test campaign has been conducted and acoustic as well as vibrational data have been acquired as these latter need to be used within the correction procedure. It has been demonstrated that in the specific rail configuration, the exceeding noise due to the rail roughness was about $6 \mathrm{~dB}(\mathrm{~A})$.

From presented data is so evident the importance of keeping into account the rail rail parameters as they may strongly influence the emitted noise with severe consequence over the acceptance vehicle test.

\section{References}

1. Thompson, D.J., Wheel-Rail Noise Generation, Part I: Introduction and Interaction Model, Journal of Sound and Vibration,161, 1993a.

2. Thompson, D.J., Wheel-Rail Noise Generation, Part II: Wheel Vibration, Journal of Sound and Vibration,161, 1993 b.

3. Thompson, D.J., Wheel-Rail Noise Generation, Part I: Inclusion of wheel rotation, Journal of Sound and Vibration, 161, 1993c.

4. Thompson, D.J., Experimental Analysis of Wave Propagation in Railway Propagation in Railway Track, Journal of Sound and Vibration, 203(5), 1997.

5. James T. Nelson, Transit Cooperative Research Program - Wheel/Rail Noise Control Manual TCRP report 23.

6. Ente Italiano di Normazione, UNI/CT 050 , FprCEN/TR 16891 "Railway applications - Acoustics Measurement method for combined roughness, track decay rates and transfer functions".

7. Viscardi M., Napolitano P., Ferraiuolo S. - An Innovative Procedure for the Rolling Noise Evaluation (2016) MATEC Web of Conferences, 76, art. no. 05013 Doi. 10.1051/matecconf/20167605013

8. Siano D., Viscardi M., Panza, M.A., Acoustic Optimization of a High-speed Train Composite Sandwich Panel Based on Analytical and Experimental Transmission Loss Evaluation Integrated by FE/Test Correlation Analysis Energy Procedia 81:689-703 . December 2015 DOI: 10.1016/j.egypro.2015.12.075

9. Viscardi, M., Napolitano, P., Arena, M. -Simulation and experimental validation of fatigue endurance limit of copper alloy for industrial applications (2016) International Journal of Mathematical Models and Methods in Applied Sciences, 10, pp. 340-346

10. Viscardi M., Rusciano N., Iadevaia M., Siano D. - An optimization process experience for HVAC noise emission and flows distribution inside a passenger's train wagon (2009) 16th International Congress on Sound and Vibration 2009, ICSV 2009, 6, pp. 3334-3341.

11. Massimo Viscardi, Pasquale Napolitano and Maurizio Arena - An innovative numerical approach for railway rolling noise forecast (2017)- Proceedings of the 24th International Congress on Sound And Vibrations ICSV24 